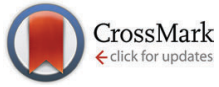

Cite this: J. Mater. Chem. C, 2016 4,8670

Received 22nd June 2016

Accepted 16th August 2016

DOI: $10.1039 / c 6 t c 02587 k$

www.rsc.org/MaterialsC

\title{
Microfluidic synthesis of micrometer-sized photoresponsive actuators based on liquid crystalline elastomers $\dagger$
}

\author{
L. B. Braun, T. Hessberger and R. Zentel*
}

\begin{abstract}
The fabrication of photoresponsive micrometer-sized liquid crystalline elastomer (LCE) particles has been described in this article for the first time. The preparation of the LCE particles using a microfluidic device by a continuous "on the fly" technique allows their fast processing with an irradiation time of less than 2 seconds. In order to accelerate the polymerization and to make the preparation of samples with thicknesses of several hundred microns possible, we modified a NIR-photoinitiation system by adding a bleaching agent. In addition to the photoinitiator, a side-chain liquid crystalline monomer containing an azo-group and a LC-crosslinker were used in the polymerization mixture. For the optimization of the polymerization parameters, LCE films were produced first. These films showed a strong thermoresponsive actuation of more than $40 \%$ upon the nematic-isotropic phase transition and changed their length by up to $34 \%$ during irradiation with light for just 8 seconds, due to the trans-cis isomerization of the azo-group. Fabricated particles were investigated with respect to their response to heating, irradiation with light and swelling in a good solvent. A strong photoresponsive actuation of about $24 \%$ was found for these particles, which was reversible either by irradiation with red light ( 8 minutes) or by an isothermal back-isomerization of the azo-group in the dark (17 minutes). The reversibility of the light induced actuation of our particles makes them promising candidates for application as artificial muscles in microrobotics.
\end{abstract}

\section{Introduction}

Liquid crystalline elastomers (LCEs) attract attention due to their actuation properties, which were already predicted by de Gennes $^{1}$ in 1975. They are the subject of many articles and books and are promising candidates for application as artificial muscles in microrobotics. ${ }^{2-10}$ These materials combine the entropy elasticity of an elastomer with the self-organization of a liquid crystal in which the latter acts as an anisotropic solvent for the isotropic polymer chain. In LCEs the mesogens of the liquid crystal either are part of the polymer chain (main-chain LCEs) or they are attached via an alkyl spacer (side-chain LCEs) to provide a good interaction. Due to the orientation of the mesogens the polymer chain is deformed into a stretched conformation below the clearing temperature, but when the sample is heated into the isotropic phase the order is lost and the polymer backbone can relax into the random coil conformation. If the liquid crystalline phase is present as a monodomain, the phase transition results in a macroscopic change in the shape of

Institute of Organic Chemistry, Johannes Gutenberg-Universität Mainz,

D-55099 Mainz, Germany. E-mail: zentel@uni-mainz.de

$\dagger$ Electronic supplementary information (ESI) available. See DOI: 10.1039/c6tc02587k the whole sample. That is, it expands in a certain direction and contracts in the opposite. With main-chain LCEs, actuations of up to $400 \%$ could be obtained ${ }^{11}$ and thus much more than what is achievable with piezo-actuators. Also compared to high actuating hydrogels, they have the advantage that no mass transfer is necessary. To gain a monodomain within the LCE, an application of an external force is necessary for the alignment of the single domains before crosslinking. This can be for example: stretching of the prepolymerized sample,$^{12}$ aligning by magnetic or electric fields ${ }^{13,14}$ and orientation with the aid of a photo-alignment layer..$^{15}$ For the orientation of the mesogens in droplets the flow in a high viscous oil in microfluidic devices is the best known way. ${ }^{16,17}$

So far thermoactuating LCEs, which change their shape (expand and contract) by heating them above the clearing temperature, are investigated mostly. However, a response to a temperature change of the environment is not desirable for devices, because then the whole surrounding needs to be heated up. So actuation induced in a more practical way is desired. Zappe et al. solved this problem by incorporating a thin platinum wire into a radial oriented LCE disc. ${ }^{9}$ In this manner they could heat the sample locally with electricity. They used this local stimulus to imitate the movement of a human iris. ${ }^{10}$ 
Magnetic and electric fields can also be used to trigger LCE actuators, but make the addition of inorganic materials necessary. ${ }^{18-21}$

Great attention has also been paid to photoresponsive LCEs. Electromagnetic irradiation can here be used in two ways. At first, radiation can be used for local heating caused by light absorption $^{22-25}$ or for a chemical modification of the LC-material by photoisomerization (isothermal shift of the phase transition temperatures due to the creation of less mesogenic compounds). In this respect LCEs containing an azo-benzene group in the mesogenic unit are attractive. In their ground state azobenzenes are present as the trans-conformer, but can be switched into the cis-isomer by continuous irradiation with UV-light. This transcis isomerization can be reversed either by thermal backisomerization or by irradiation with light of higher wavelength $(\lambda>450 \mathrm{~nm}) .{ }^{26}$ While the trans-isomer has a rod-like shape and is well compatible with LC-phases, the cis-isomer is bent, whereby the liquid crystalline order is reduced. This results in a decrease of the order parameter $S$ and the clearing temperature. Thus, during photoisomerization of an azobenzene containing LCE sample, actuation can be observed (Fig. 1).

While the thermal back-isomerization for azobenzenes in isotropic solution usually takes several hours, it could be shown in former studies that this process duration is reduced to several minutes by incorporating them into a liquid crystalline matrix. $^{27,28}$ This faster switching behavior makes azobenzenes even better candidates for use in photoresponsive LCEs and many actuators were prepared already from them. Of course the rate of the switching between trans- and cis-forms of azobenzenes can also be changed by modifying the nature of the chromophoric system. ${ }^{29,30}$ However, so far, mostly densely crosslinked systems, which work in the bending mode to obtain large macroscopic deformations, were investigated ${ }^{31-34}$ and little attention was paid to the expansion/contraction associated with the phase transition. The group of Ikeda produced a light driven engine for example, ${ }^{35}$ Fischer et al. fabricated biomimetic photoresponsive swimmers ${ }^{36}$ and the group of Broer synthesized artificial cilia, which bend during UV-irradiation. ${ }^{37}$

We were interested in the fabrication of photoactuating LCEmicro-particles, which were to be produced in a microfluidic capillary based device, and the investigation of their lightresponsive properties. To prepare these particles it became necessary to establish a highly efficient photopolymerization of the azo containing monomer mixture with an optimized NIR-initiator system. With the optimized monomer mixture, particles could then be produced, which showed a thermoresponsive actuation of up to $33 \%$ and a fast elongation of $24 \%$

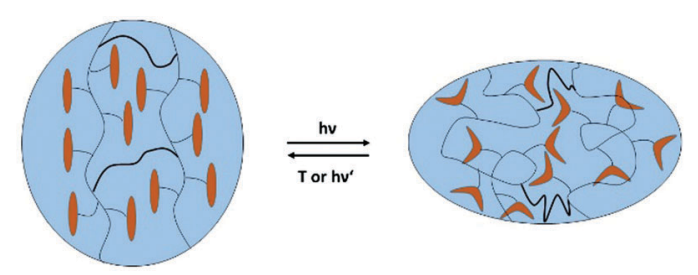

Fig. 1 Schematic drawing of the photoactuation of an azo-LCE. during irradiation in just a few seconds. Besides a thermal back-deformation we also show that a back-deformation induced by red-light could be achieved. In addition, the swelling behavior of the particles was investigated as a third way of actuation.

\section{Results and discussion}

\section{Selection of the initiator system}

For the polymerization in a microfluidic device the initiator has to fulfill several criteria. On the one hand a fast curing of the monomer droplets is necessary, so that solid (cross-linked) particles leave the device, while on the other hand the polymerization must not be started prior to droplet formation to prevent clogging of the capillary and tubes. Furthermore, the solid monomer mixture needs to be heated into the liquid crystalline phase before polymerization which prohibits the application of thermally induced initiators. One possible way to meet these requirements is a redox-initiated polymerization, in which both redox components come into contact only during droplet formation. ${ }^{38}$ A more convenient solution is the use of photoinitiators. Usually UV-photoinitiators are used for this kind of polymerization, but as azobenzenes absorb light strongly in the UV- and blue range of the spectrum, no efficient curing of the mixture is possible. This is, however, necessary, because the particles remain for only a short time in the capillary. In addition, trans-cis isomerization takes place when azobenzenes are irradiated with UV-light. Thus, the absorption of UV-light might result in a loss of the liquid crystalline phase during the polymerization. To overcome these problems we used $1,3,3,1^{\prime}, 3^{\prime}, 3^{\prime}$ hexamethyl-11-chloro-10,12-propylene-tricarbocyanine triphenylbutyl borate (CBC, Fig. 2b) as an initiator which was already described by Keller et al. for the synthesis of LCE films made out of the same monomer. ${ }^{39,40}$ Having an absorption maximum of $\lambda=780 \mathrm{~nm}$ this molecule starts the polymerization by NIRirradiation. Thus, the wavelength is high enough not to be absorbed by the monomer (Fig. 2a) and no trans-cis isomerization occurs. As this initiator is already very reactive under dimmed room light, we synthesized it in situ (see the Experimental section).

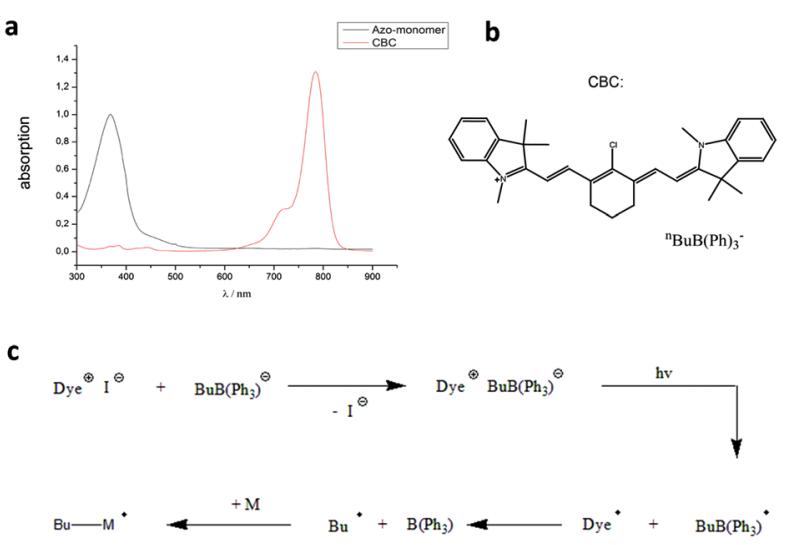

Fig. 2 (a) UV-Vis spectrum of CBC compared to that of the azo-monomer, (b) chemical structure of $\mathrm{CBC}$ and (c) assumed mechanism of the initiation step. 
Thus, the ion pair of the initiator forms in the reaction mixture and starts the polymerization when the mixture is irradiated with intense NIR/red light. In addition borate $\mathrm{V}$ also works as a bleaching agent for the dye so that even thick samples can be cured. The assumed mechanism ${ }^{41}$ for the initiation step is shown in Fig. 2c.

\section{Preparation of particles using a microfluidic device}

For the fabrication of our photoresponsive LCE particles we used a capillary based microfluidic setup that was already applied for the production of thermoresponsive LCE particles in our group and is shown in Fig. 3a. ${ }^{42,43}$

In our device the monomer mixture flew through a thin glass capillary, which was positioned along the main axis of a T-junction, while a high viscous continuous phase (we used a silicon oil with a viscosity of $1000 \mathrm{cSt}$ ) was pumped through the perpendicular entrance of the T-junction and flew round the capillary. Due to the high share rates at the capillary's tip, droplets were formed and flew down the polymerization tube (Fig. 3a). For a good control over the flowrates of continuous and monomer phases (dispersed phase) syringe pumps were used to pump the fluids through the capillaries and tubes. As the monomer, we chose a side-on liquid crystalline azo-monomer with the phase behavior (crystalline $53{ }^{\circ} \mathrm{C}$ nematic $84{ }^{\circ} \mathrm{C}$ isotropic), which was first synthesized in the group of Keller $^{44}$ and was already applied for the preparation of photoactuating films. Besides the monomer the dispersed phase consisted of $10 \mathrm{~mol} \%$ of a liquid crystalline crosslinker (Fig. 3d), $1.4 \mathrm{wt} \%$ of the initiator dye and $3 \mathrm{wt} \%$ of Borate $\mathrm{V}$. The resulting mixture showed a nematic phase with a clearing temperature of $72{ }^{\circ} \mathrm{C}$, but the crystals did not melt before they were heated up to $85{ }^{\circ} \mathrm{C}$. This made a modification of the microfluidic device necessary. The monomer mixture was placed in the tubing from the syringe pump to the T-junction and was pumped through the device by a low viscous silicone oil provided by a syringe. Both the tubing and the T-junction were placed in a water bath which was heated to $90{ }^{\circ} \mathrm{C}$ so that the monomer mixture was present as an isotropic liquid. The polymerization tube, after leaving the water bath, was laid on a hotplate that was heated to $65{ }^{\circ} \mathrm{C}$. Thus, the monomer droplets were cooled into the nematic phase before they were polymerized using a HighPower LED $(\lambda=623 \mathrm{~nm})$. In the running set-up the droplets typically flew less than 2 seconds under the light spot.

For photoinitiation with visible light we chose the initiation system presented in Fig. $2 \mathrm{~b}$ and reported by Keller et al. in ref. 40. However the system did - at first - not work effectively under the short time span of the microfluidic experiment. Thus, we increased the amount of Borate $\mathrm{V}$ (butyryl choline butyltriphenylborate), which also acts as a clearing agent, by a factor of about 2. With this initiator system the irradiation time of 2 seconds was sufficient to decompose the initiator, as could be seen by the disappearance of the initiator's green color. At the end of the tubing the droplets were collected in a small glass vial and found to be solid monodisperse particles (Fig. 3b). So a rather short time period was sufficient to solidify the LC monomer droplet by VIS-initiated radical polymerization.

\section{Investigation of the best parameters for the system}

To optimize thermal and photochemical actuation of the LC-elastomers, independent of the processing parameters in the capillary, we produced films of different thicknesses and crosslinker ratios. For this purpose, a few crystals of the monomer mixture were taken on a glass plate, melted at $100{ }^{\circ} \mathrm{C}$ and a second glass plate was put on top, separated from the lower one with a spacer foil of varying sizes. Afterwards, it was cooled to $65{ }^{\circ} \mathrm{C}$ into the liquid crystalline phase and irradiated with red light $(623 \mathrm{~nm})$. During the cooling and polymerization of the sample a horseshoe magnet was put around the setup in order to achieve a good orientation of the mesogens inside the film. It was shown by the group of Zappe that magnetic fields higher than $100 \mathrm{mT}$ are sufficient for this purpose. ${ }^{10}$ For the determination of the actuation we cut little pieces of the LCE film $(c a .500 \mu \mathrm{m} \times 200 \mu \mathrm{m})$ and observed their change in width during the heating above the clearing temperature or during irradiation for about 10 seconds (continuous wavelength at $70{ }^{\circ} \mathrm{C}$ ). The actuation was then calculated by dividing the length of the film before the deformation $\left(l_{0}\right)$ by the length afterwards $\left(l_{1}\right)$ (actuation $\left.=100 \% \times l_{0} / l_{1}\right)$.

Next we characterized the thermal parameters of the prepared liquid crystalline polymer. The ESI $\dagger$ (Fig. S5) contains a DSC a

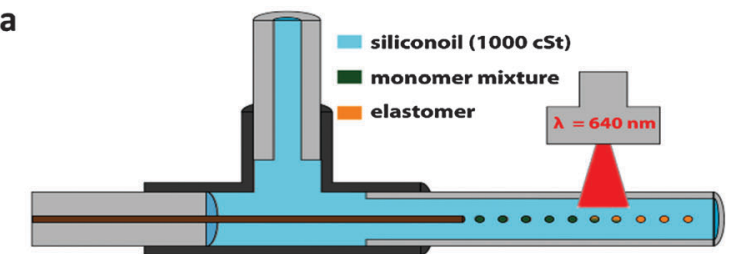

b

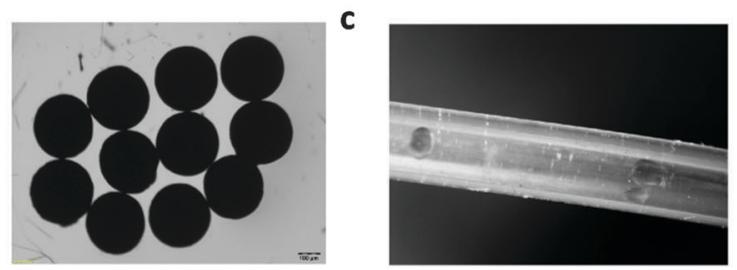

d

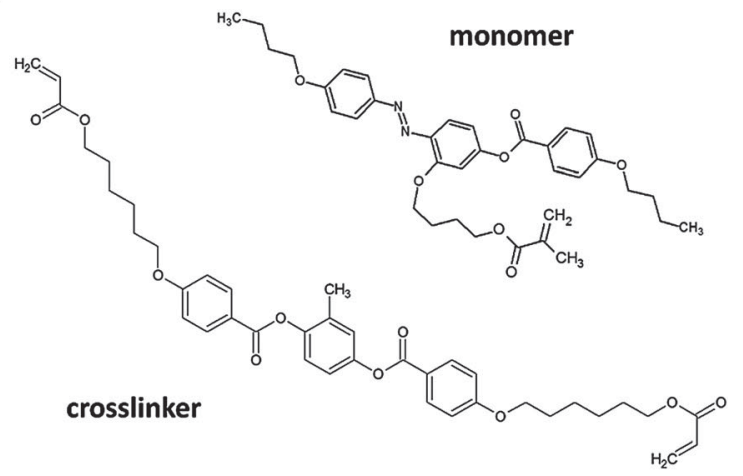

Fig. 3 (a) Schematic drawing of the microfluidic setup, (b) monodisperse LCE particles produced in our device (scale bar: $100 \mu \mathrm{m}$ ), (c) droplet formation at the tip of the capillary in the microfluidic device and (d) chemical structure of the monomer and the crosslinker. 
a

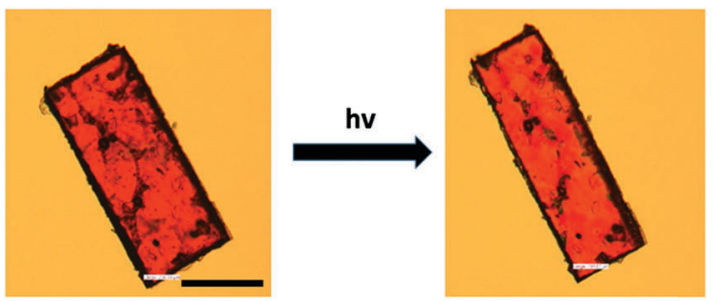

b

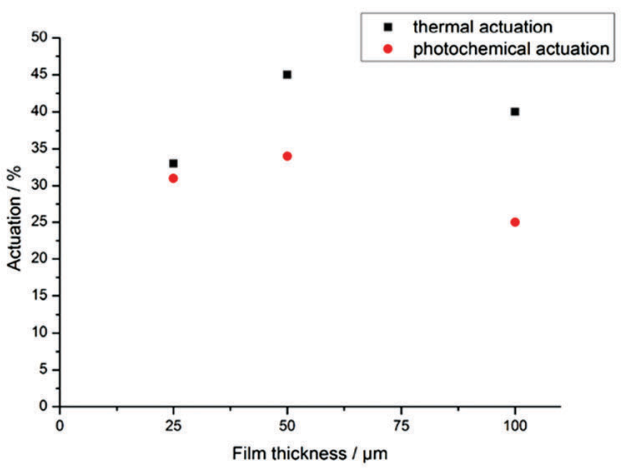

C

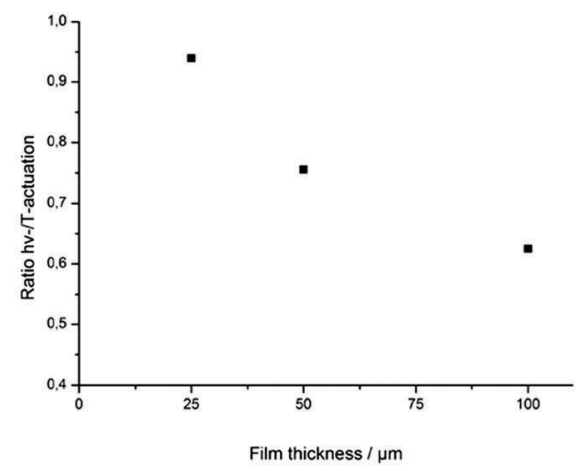

Fig. 4 (a) LCE film before and after irradiation at $70{ }^{\circ} \mathrm{C}$ (scale bar: $200 \mu \mathrm{m}$ ), and (b) influence of the film thickness on the actuation and (c) on the ratio between photochemical and thermal actuation (irradiation for the photochemical actuation in (b) and (c) was performed at $70{ }^{\circ} \mathrm{C}$ again).

measurement of the polymer prepared. It shows a $T_{\mathrm{g}}$-value of $37{ }^{\circ} \mathrm{C}$ and a clearing temperature (nematic-isotropic) at $90{ }^{\circ} \mathrm{C}$. Thus above $40{ }^{\circ} \mathrm{C}$ (or more realistically $50{ }^{\circ} \mathrm{C}$ ) the crosslinked polymer is in the elastomeric state, and that is where nearly all measurements were done.

One of the actuating films $\left(70{ }^{\circ} \mathrm{C}\right)$ is shown before and after irradiation in Fig. $4 \mathrm{a}$ and also a video of this sample is available in the $\mathrm{ESI} \dagger$ (S1). For the films, a maximum thermal actuation of $45 \%$ and a maximal photo-actuation of $34 \%$ by irradiation with a white-light LED (the spectrum is shown in the ESI $\dagger$ (S3)) about $15{ }^{\circ} \mathrm{C}$ below the clearing temperature could be achieved. This is, to the best of our knowledge, the strongest response to light of azo-LCEs published in a regular journal till now.

The results for the films of different thicknesses are shown in Fig. $4 \mathrm{~b}$ and c. For very thin films of just $25 \mu \mathrm{m}$ thickness thermal actuation is not as good as for the samples with 50 and $100 \mu \mathrm{m}$ thickness. This is due to a slight rolling of the thin films, which makes actuation less effective. With thermal actuations around and above $40 \%$ the actuation is in the typically range of values for LCEs from side-on fixed mesogens. ${ }^{2}$

The photo-induced actuation for samples - about $15{ }^{\circ} \mathrm{C}$ below the clearing temperature of the all trans system - is in a comparable range, but always lower than thermally actuated systems. This is reasonable as trans to cis isomerization induces in these samples a nearly isotropization of the liquid crystalline phase. Here the achievable actuation is maximal for films of $50 \mu \mathrm{m}$ thickness and decreasing for thinner and thicker films. For the thin film again slight rolling is observed; for the thicker sample the reduction in actuation is a consequence of the longer distance the irradiation has to take. This reduces the UV-intensity along the path and results in a less strong change in the shape for parts of the sample.

The UV-Vis spectrum of the monomer in solution - before and after irradiation with the white light LED - is shown in Fig. 5. By continuous excitation of the monomer with our lamp the molecules undergo trans-cis isomerization, which can be seen by the decrease of the $\pi \rightarrow \pi^{*}$-band (around $365 \mathrm{~nm}$ ) of the trans-azobenzene. At the same time the $\mathrm{n} \rightarrow \pi^{*}$ band ( $>450 \mathrm{~nm}$ ) of the cis-conformer increases slightly. Thus, by irradiation with red light the photochemical isomerization of the azo-monomer can be reversed.

Changing the content of the crosslinker in the mixture has a big influence on maximal actuation (Fig. 6). For small amounts of up to $25 \mathrm{~mol} \%$ the actuation is - with values over $40 \%$ - quite stable, but for a high concentration of $50 \mathrm{~mol} \%$ the deformation of the film is much smaller. With a higher crosslinker concentration the whole film becomes much stiffer and that is probably the reason for a weaker actuation.

For higher crosslinker concentrations also the ratio between the photochemical and thermal actuation decreases strongly. This is logical as the crosslinker does not bear an azo-group and thus cannot contribute to increasing the disorder by bending.

By investigating the parameters of the photoactive LCE films we not only noticed that our system has a stronger photoresponsive actuation than conventional systems, but also has the advantage of showing a fast response to light (full actuation in less than 10 seconds) and moreover is polymerizable in thicker films, a property that is inalienable for the production of particles. In previous works the photopolymerization was

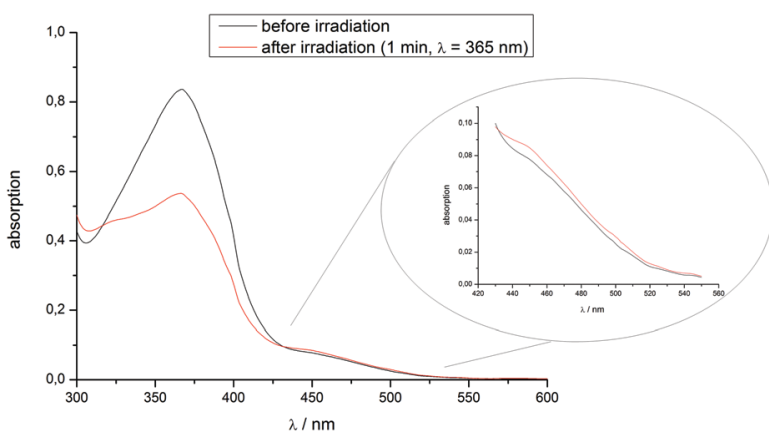

Fig. 5 UV-Vis spectrum of the azo-monomer in solution, before and after irradiation with the white light LED for 1 minute. 


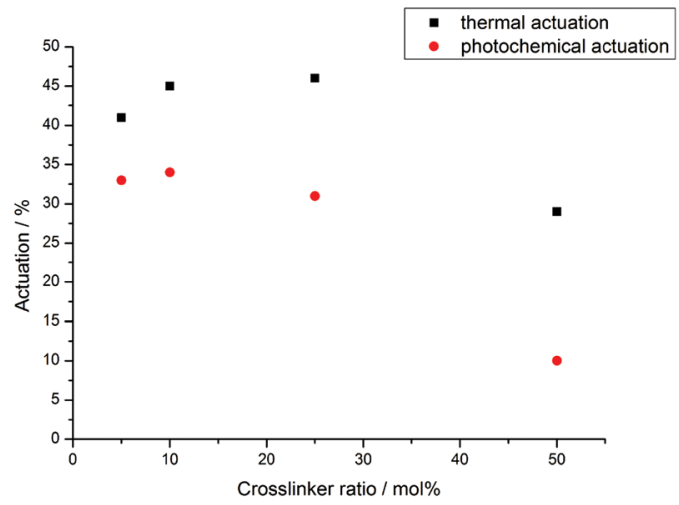

b

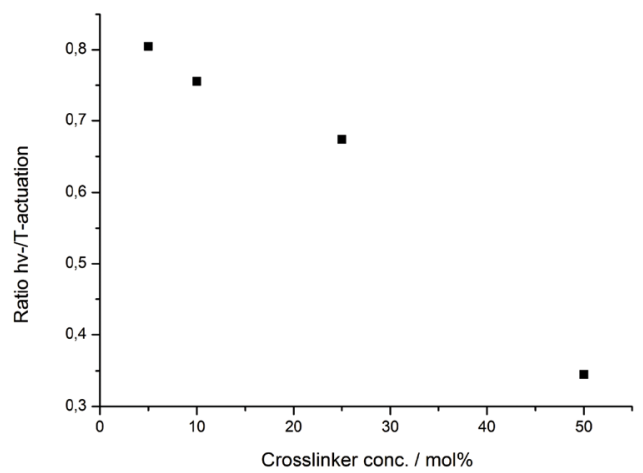

Fig. 6 (a) Influence of the crosslinker concentration on the actuation properties of the film and (b) on the ratio between photochemical and thermal actuation (irradiation for the photochemical actuation in (a) and (b) was performed at $70{ }^{\circ} \mathrm{C}$ ).

often limited to a sample thickness of a few micrometers, but by adding a higher concentration of the bleaching agent borate $\mathrm{V}$ to the initiator mixture this problem could be overcome, as the initiator got decolored during irradiation.

Another remarkable quality of our films is that they show no bending during irradiation, which is the typical response of densely crosslinked azosystems. ${ }^{31,32}$ Instead they deform uniaxially. This is a big advantage for use in devices since a distortion along just two axes enables the application of distinct higher forces compared to three dimensional bending.

\section{Results of the particle fabrication}

Monodisperse LCE particles with diameters of 300-500 $\mu \mathrm{m}$ were produced as described above and then investigated on a precise heating plate under a polarized light microscope. To examine the photoresponsive behavior the particles were heated to a fixed temperature close to the clearing temperature of the all-trans sample and then irradiated with a cold-light source (continuous spectrum) to prevent additional warming of the particles. The irradiation was focused by two gooseneck light guides, giving a spot of about $0.5 \mathrm{~cm}$ diameter. As a result of their preparation conditions these LCEparticles have a slightly pumpkin like shape and extend along their short axis (see ref. 16). The mesogens in these particles are aligned along a concentric orientation field within the particles with the centers of the concentric rings lying on the axis through the poles. a

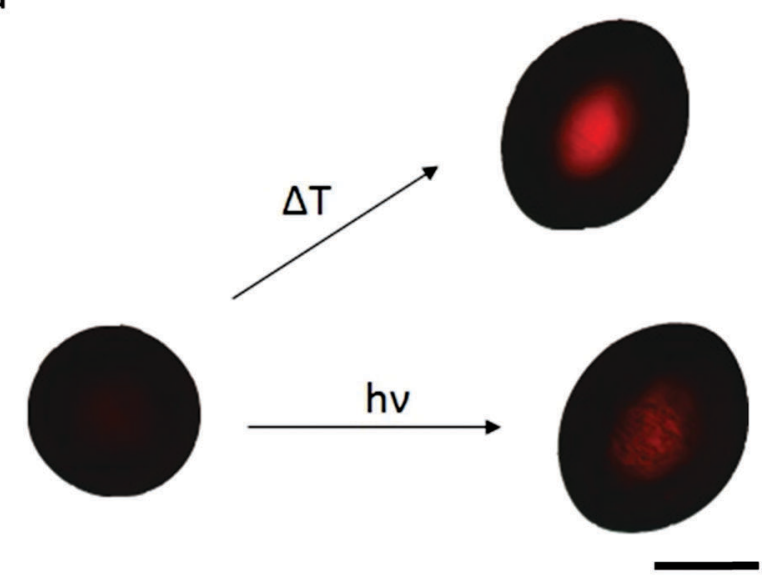

b

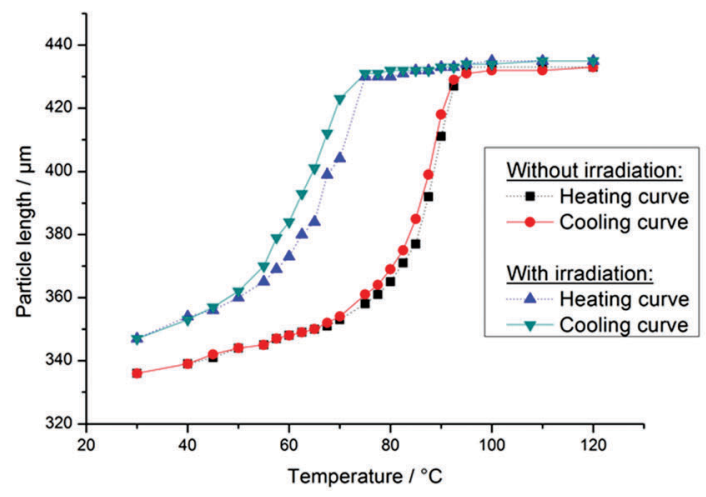

C

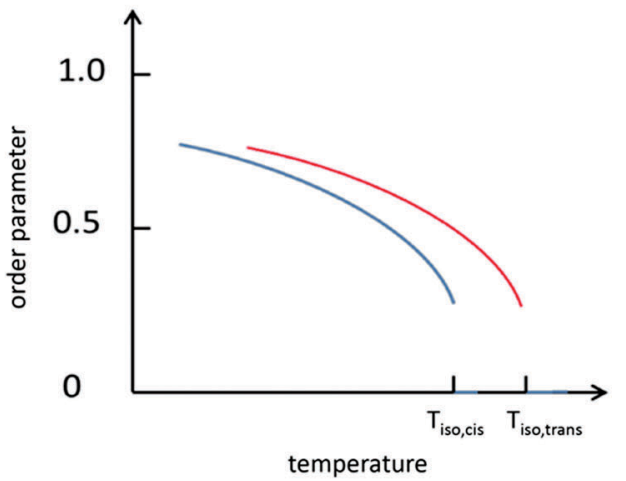

Fig. 7 (a) The two ways of inducing a phase transition and thus a deformation of the particle by either heating above the clearing temperature of the particle or by irradiation at $70{ }^{\circ} \mathrm{C}$ with light of a continuous spectrum (scale bar: $200 \mu \mathrm{m}$ ), (b) thermoresponsive behavior of the particle diameter under and without irradiation and (c) schematic drawing of the order parameter's temperature dependence for the LCE with and without irradiation.

Fig. 7a shows the response of one particle to both stimuli. In either case the LCE exhibits a nematic phase at $70{ }^{\circ} \mathrm{C}$ before it attains the isotropic phase by the application of the external stimulus. According to the concentric orientation of the mesogens the particle elongates along the axis of its poles during the phase transition and shrinks in the perpendicular direction. The bright spot in the center of the thermally actuated particle indicates a 
complete phase transition. Compared to the LCE films the particles showed a smaller change in size, but with a thermal response of up to $33 \%$ and a photoresponsive actuation of up to $24 \%$ it was still strong. For the latter a video is also available in the ESI $\dagger(\mathrm{S} 2)$.

The thermoresponsive length change of the particles is shown in Fig. $7 \mathrm{~b}$ and according to the literature ${ }^{42}$ an s-shaped curve is obtained showing a hysteresis for the heating and cooling curve. Starting from low temperatures the order parameter $S$ of the mesogens in the LCE is expected to get reduced more and more leading to a slight increase of the particle length (see the schematic drawing in Fig. 7c). At the clearing temperature, finally, a drastic loss of orientation occurs ( $S$ becomes zero) so that the length of the particles changes strongly by just heating them up a few degrees. Once the LCE is in its isotropic state no further elongation can be observed. By cooling the particle the s-shaped curve is retained and only very slightly shifted to lower temperatures. Furthermore, it can be seen that the hysteresis curve for a particle under irradiation is shifted about $15{ }^{\circ} \mathrm{C}$ to lower temperatures. This is a consequence of the lowering of the clearing temperature of the liquid crystalline phase by about $15{ }^{\circ} \mathrm{C}$, induced by the cis-isomer created by photoisomerization. The same is valid for the order parameter of the liquid crystalline phase inside the LCE (Fig. 7c). So if the sample in the all-trans (dark) state is kept at a temperature not more than $15{ }^{\circ} \mathrm{C}$ below the clearing temperature, isotropization and thus a full shape change should be achievable by UV-irradiation (see Fig. 7b).

Also the photoresponsive behavior of the particles was investigated and is shown in Fig. 8. Part a illustrates the photoresponsive actuation as a function of time. By irradiation with the cold-light source at $70{ }^{\circ} \mathrm{C}$ the particle elongates linearly before the actuation is completed after about 7 seconds. Then, no further change in the length can be observed for a longer irradiation time. After the light source is shut off the azo-mesogens undergo a thermal back-isomerization to the trans-conformer and thus the liquid crystalline phase is formed again which results in a backdeformation of the particle. This return to the original shape takes about 16 minutes at $70{ }^{\circ} \mathrm{C}$ as it can be seen in Fig. 7b. As mentioned before, the back-isomerization to the trans-form can also be triggered by an excitation of the cis-conformer's $n \rightarrow \pi^{*}$ band with red light and thus, the necessary time for the backdeformation can be halved to 8 minutes at the same temperature. Here also the cold-light source was used for the irradiation, but with a red light filter (for the transmittance spectrum see S4, ESI $\dagger$ ) being held between the lamp and the sample. We also tried to induce back-deformation by the irradiation of the particle with light of a wavelength around the maximum of the $n \rightarrow \pi^{*}$-band (450 $\mathrm{nm})$, but surprisingly no back-deformation could be seen at this wavelength. This observation was also made by the group of Keller, who experienced the same result. ${ }^{40}$ We assume that this is due to an equal absorption of the trans- and cis-conformer at this wavelength. In contrast at wavelengths above $550 \mathrm{~nm}$ the absorption of the cis-form is indeed weak but probably stronger than the absorption of the trans-form. In part (c) of the figure the actuation is shown as a function of temperature during the irradiation. trans-cis isomerization in photodynamic equilibrium a

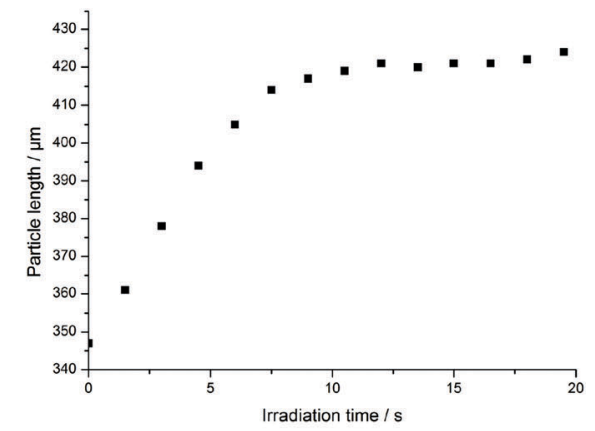

b
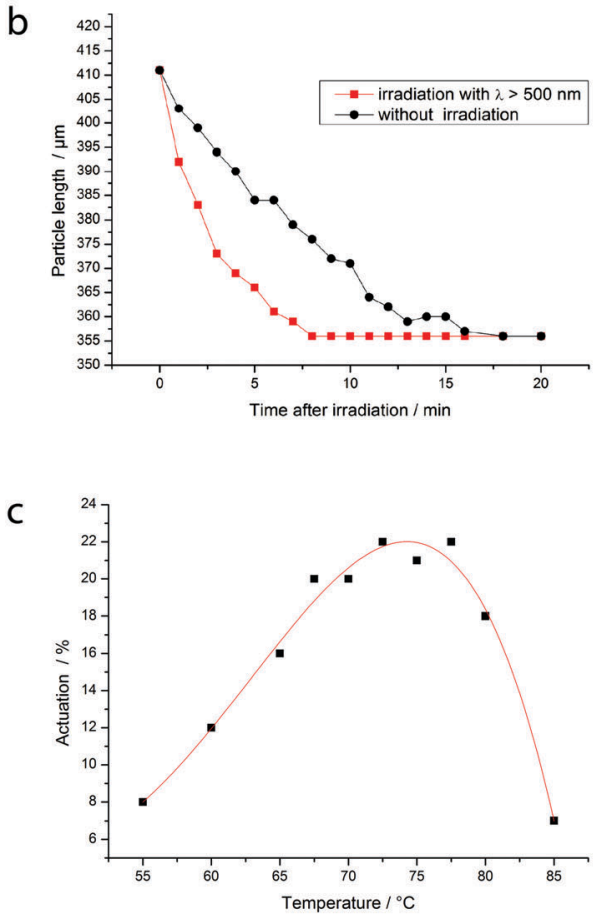

Fig. 8 Photoresponsive behavior of the LCE particles. (a) Actuation as a function of time (at $70{ }^{\circ} \mathrm{C}$ ), (b) thermal and photochemically induced back deformation as a function of time (also at $70^{\circ} \mathrm{C}$ ) and (c) the photoresponsive actuation for different temperatures during irradiation.

as a result of the irradiation leads in the neat nematic phase of our system only to a decrease of the clearing temperature. Thus, at temperatures far below the clearing point just a reduction of the mesogens' order can be achieved which engenders just a small deformation of the LCE. Hence, the actuation increases the closer the temperature is to the clearing temperature during irradiation. Very close to the clearing temperature of the all-trans system much of the deformation has already been done in a thermally actuated manner. Thus the contribution of photoactuation gets small again. At intermediate temperatures the effect of photoactuation is maximal. These results match well with the observations of Fig. 7.

In conclusion it could be shown that a fast and remarkable photoactuation can be achieved by irradiation of our LCEs at temperatures close to the clearing point. Drawbacks are the distinctly slower back-deformation and that no actuation at room temperature can be achieved with this system. 


\section{Swelling of the particles as a third stimulus}

Beside the thermal and photochemical actuation, swelling in a good solvent can also destroy the liquid crystalline phase inside the particle and thus induce anisotropic deformation. As shown in Fig. 9 the crosslinked LCE-particles do not just grow to a multiple of their original size, but also elongate along the same axis - as if they were heated up - when they are swollen in chloroform. Once the solvent around the particle is evaporated the particle shrinks again. However at room temperature they do not relax back to their original shape. This occurs probably because the material of the shell of the particle is too close to the glass transition temperature and hard, when the interior of the particle turns back to the nematic phase due to evaporation of all the solvent. That is the particle as a whole lacks mobility to adopt the original shape.

By heating the particle to $110{ }^{\circ} \mathrm{C}$ the mobility to reach the thermodynamically favored conformation is regained, but as the sample is now in the isotropic state it remains elongated. But by cooling the particle slowly down to room temperature, it regains its original shape.

\section{Experimental section}

\section{Materials and reagents}

Chloroform, dichloromethane and silicone oil (100 cSt and $1000 \mathrm{cSt}$ ) were purchased from Sigma-Aldrich. Butyryl choline butyltriphenylborate (Borat V) was purchased from Spectra group limited. The liquid crystalline monomer (4-butoxy-2'-(4methacryloyloxybutoxy)-4'-(4-butoxybenzoyloxy)azobenzene) ${ }^{45}$ the crosslinker (1,4-bis (4-(6'-acryloxy-hexyloxy)benzoyloxy)-2-toluene $)^{46}$ and the dye for the initiator $\left(1,3,3,1^{\prime}, 3^{\prime}, 3^{\prime}\right.$-hexamethyl-11-chloro10,12-propylene-tricarbocyanine iodide) ${ }^{41}$ were synthesized according to the literature. Components for the microfluidic device were obtained from WICOM (PTFE tubings) and Postnova Analytics GmbH (fused silica capillary, T-junction, nuts and ferrules).

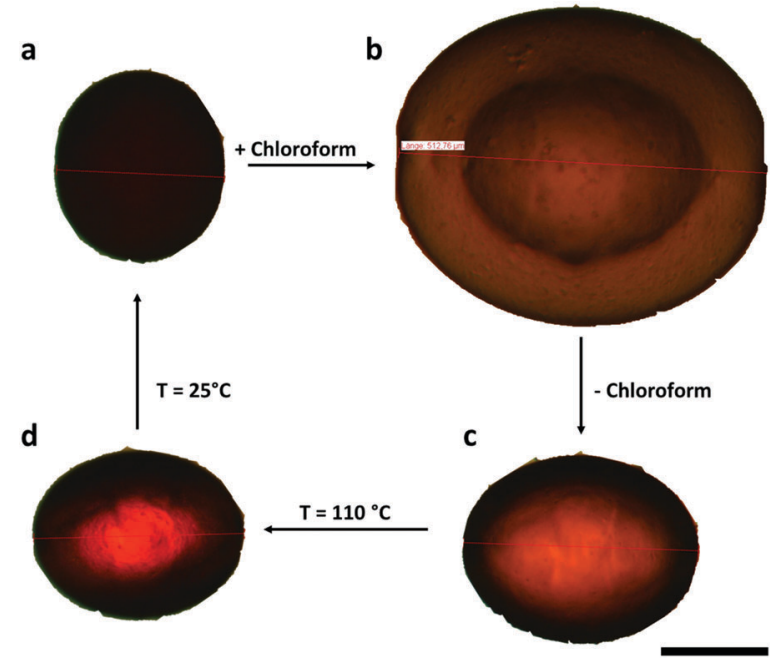

Fig. 9 Swelling behavior of the LCE particles: (a) before any treatment, (b) after swelling in chloroform, (c) after evaporation of surrounding chloroform and (d) after heating of the particle to $110{ }^{\circ} \mathrm{C}$ (scale bar: $200 \mu \mathrm{m}$ ).
A red light HighPower LED (623 nm) was purchased from Conrad and the coldlight source KL 1600 (spectrum see S3, ESI $\dagger$ ) from Schott.

\section{Preparation of polymer films}

The monomer mixture used for the film syntheses was prepared by mixing the monomer, the crosslinker $(10 \mathrm{wt} \%$, except for the experiments with varying crosslinker concentrations), $1.4 \mathrm{wt} \%$ 1,3,3,1', $3^{\prime}, 3^{\prime}$-hexamethyl-11-chloro-10,12-propylene-tricarbocyanine iodide and $3 \mathrm{wt} \%$ Borate $\mathrm{V}$ in dichloromethane. The solvent was removed under vacuum. A few crystals of the monomer mixture were melted at $100{ }^{\circ} \mathrm{C}$ on a glass slide which was laid on a precision hot plate (Harry Gerstig $\mathrm{GmbH}$ ) in the magnetic field of a horseshoe magnet $(B>100 \mathrm{mT})$. A second glass slide was put on top, separated from the lower one with spacer foil of varying sizes ( $25 \mu \mathrm{m}, 50 \mu \mathrm{m}, 100 \mu \mathrm{m}$ and $250 \mu \mathrm{m}$ ). Then the mixture was cooled to $65{ }^{\circ} \mathrm{C}$ into the liquid crystalline phase and irradiated with red light $(623 \mathrm{~nm})$ for about 5 seconds. After cooling to room temperature the glass slides were separated with a scalpel, with the film just sticking to one of them. For further investigation, we cut little pieces out of the LCE film (ca. $500 \mu \mathrm{m} \times 200 \mu \mathrm{m})$.

\section{Preparation of the particles}

Fig. 3a shows a schematic drawing of the microfluidic setup. A glass capillary (inner diameter (ID): $100 \mu \mathrm{m}$ ) was inserted inside a T-junction along its main axis. The capillary's tip, where the droplets are formed, ended in an outlet polytetrafluoroethylene (PTFE) tubing (ID: $750 \mu \mathrm{m}$ ) and the continuous phase (silicone oil, $1000 \mathrm{cSt}$ ) was provided by a tubing of the same material (ID: $500 \mu \mathrm{m}$ ) through the perpendicular entrance of the T-junction. For a good control over the flow rate, a syringe pump (Pump 33, Harvard Apparatus) was used to pump the monomer phase at stable flow rates through the setup. The capillary, which provided the monomer mixture, began in another PTFE tubing (ID: $170 \mu \mathrm{m}$ ) on the side of the T-junction opposite to the polymerization tube. Besides the syringe pump, the setup was placed in a water bath that was kept at $90{ }^{\circ} \mathrm{C}$. Between the syringe and the capillary a piece of a PTFE tube (ID: 1/16 inch) filled with the monomer mixture was plugged, so that the monomer mixture was present as a liquid. To pump it through the capillary a low viscous silicone oil $(100 \mathrm{cSt})$ was used via the syringe. The polymerization tube was laid on a precision hot plate that was heated to $65{ }^{\circ} \mathrm{C}$. Thus, the monomer droplets were cooled down into the nematic phase before they were polymerized by the light of a HighPower red light LED (623 nm) "on the fly" inside the tube (time under the light spot: ca. 2 seconds). After passing the polymerization tube (length: $30 \mathrm{~cm}$ ) the crosslinked particles were collected in a small glass vial.

\section{Characterization}

The droplet formation at the tip of the capillary was observed using a Zeiss stereomicroscope Stemi 2000-C equipped with an Olympus XM 10 camera. For the investigation of the actuation of films and particles, we used the light microscope Olympus BX51 equipped with a hot-stage (Linkam TMS 94) and the camera Olympus ColorView II. The sizes of the samples were 
analysed using the imaging software Cell^ ${ }^{\wedge} \mathrm{D}$. For the irradiation experiments, the sample was heated to the desired temperature and then irradiated with the light of a white-light LED of the coldlight source KL 1600. Light guides were used to focus the irradiation upon the sample giving a light spot with a diameter of about $1 \mathrm{~cm}$. For the actuation of the samples (trans-cis isomerization) the light source was used without the use of any filters and for the backactuation (cis-trans isomerization) the same lamp was used, but with a red filter (OG 590 Schott, for the absorption spectrum see S4, ESI $\dagger$ ) being held in the light beam. UV-Vis spectra were recorded using a Jasco spectrophotometer V-630 in dichloromethane.

\section{Conclusions}

For the first time micrometer-sized LCE particles showing a photoresponsive deformation could be synthesized. A microfluidic device could be used to fabricate a high number of these monodisperse actuators in a continuous flow by photopolymerization with red light. For the investigation of the best fabrication parameters LCE films were also produced. These samples showed a thermoresponsive actuation of more than $40 \%$ and a fast response of $34 \%$ in length change by irradiation with light of continuous wavelength. This is, to the best of our knowledge, the strongest light induced length change (deformation) of azo-LCEs published in a regular journal so far. Even films of several hundred micron thicknesses could be produced by using a bleaching agent in the initiator system. It was remarkable that the films did not show bending but a uniaxial deformation. The then produced particles were examined with respect to their photo- and thermoresponsive behavior. It could be shown that the particles deformed when they were heated above the clearing temperature or when they were irradiated with light of continuous wavelength at temperatures close to the phase transition. Complete light induced deformation of the particles took place in just 8 seconds and could be reversed in the dark or by irradiation with red light. In addition, swelling of the particles in a good solvent was found to be a third stimulus to actuate the particles.

With this work we contribute to an improvement and a better understanding of photoresponsive LCEs. If the drawback of a slow back deformation can be overcome this system will be a good candidate for use as artificial muscles in microrobotics.

\section{Acknowledgements}

We thank Maria Müller for the DSC measurement. The authors acknowledge funding by the German Science Foundation (DFG: Ze 230/24-1).

\section{References}

1 P. G. de Gennes, C. R. Hebd. Seances Acad. Sci., Ser. B, 1975, 281, 101.

2 M.-H. Li and P. Keller, Philos. Trans. R. Soc., A, 2006, 364, 2763-2777.
3 F. J. Davis, J. Mater. Chem., 1993, 3, 551-562.

4 E.-K. Fleischmann and R. Zentel, Angew. Chem., 2013, 125, 8972-8991.

5 C. Ohm, M. Brehmer and R. Zentel, Adv. Mater., 2010, 22, 3366-3387.

6 R. L. B. Selinger, B. L. Mbanga and J. V. Selinger, Emerg. Liq. Cryst. Technol. III, 2008, 6911, 69110A.

7 T. Bera, E. J. Freeman, J. A. McDonough, R. J. Clements, A. Aladlaan, D. W. Miller, C. Malcuit, T. Hegmann and

E. Hegmann, ACS Appl. Mater. Interfaces, 2015, 7, 14528-14535.

8 R. Zentel, Adv. Mater., 1989, 28, 1407-1415.

9 S. Petsch, R. Rix, B. Khatri, S. Schuhladen, P. Müller, R. Zentel and H. Zappe, Sens. Actuators, A, 2014, 231, 44-51.

10 S. Schuhladen, F. Preller, R. Rix, S. Petsch, R. Zentel and H. Zappe, Adv. Mater., 2014, 26, 7247-7251.

11 S. V. Ahir, A. R. Tajbakhsh and E. M. Terentjev, Adv. Funct. Mater., 2006, 16, 556-560.

12 G. H. F. Bergmann, H. Finkelmann, V. Percec and M. Y. Zhao, Macromol. Rapid Commun., 1997, 18, 353-360.

13 M. H. Li, P. Keller, J. Yang and P. A. Albouy, Adv. Mater., 2004, 16, 1922-1925.

14 M. Brehmer, R. Zentel, G. Wagenblast and K. Siemensmeyer, Macromol. Chem. Phys., 1994, 195, 1891-1904.

15 K. Ichimura, Chem. Rev., 2000, 100, 1847-1874.

16 C. Ohm, N. Kapernaum, D. Nonnenmacher, F. Giesselmann, C. Serra and R. Zentel, J. Am. Chem. Soc., 2011, 133, 5305-5311.

17 E.-K. Fleischmann, H.-L. Liang, N. Kapernaum, F. Giesselmann, J. Lagerwall and R. Zentel, Nat. Commun., 2012, 3, 1178.

18 A. Kaiser, M. Winkler, S. Krause, H. Finkelmann and A. M. Schmidt, J. Mater. Chem., 2009, 19, 538.

19 M. Chambers, H. Finkelmann, M. Remškar, A. Sánchez-Ferrer, B. Zalar and S. Žumer, J. Mater. Chem., 2009, 19, 1524-1531.

20 N. Torras, J. Esteve, A. Sánchez-ferrer and K. E. Zinoviev, J. Mater. Chem. C, 2013, 1, 5183.

21 A. Sánchez-ferrer, T. Fischl, M. Stubenrauch, A. Albrecht, H. Wurmus, M. Hoffmann and H. Finkelmann, Adv. Mater., 2011, 23, 4526-4530.

22 L. Yang, K. Setyowati, A. Li, S. Gong and J. Chen, Adv. Mater., 2008, 20, 2271-2275.

23 Y. Ji, Y. Y. Huang and E. M. Terentjev, Langmuir, 2011, 27, 13254-13260.

24 J. E. Marshall, Y. Ji, N. Torras, K. Zinoviev and E. M. Terentjev, Soft Matter, 2012, 8, 1570.

25 H. Luo, Z. Li, G. Yi, X. Zu, H. Wang, H. Huang, Y. Wang, Z. Liang and S. Zhang, Mater. Lett., 2014, 137, 385-388.

26 P. Beyer, M. Krueger, F. Giesselmann and R. Zentel, Adv. Funct. Mater., 2007, 17, 109-114.

27 T. Öge and R. Zentel, Macromol. Chem. Phys., 1996, 197, 1805-1813.

28 B. Fischer, C. Thieme, T. M. Fischer, F. Kremer, T. Oge and R. Zentel, Liq. Cryst., 1997, 22, 65-74.

29 (a) A. Sánchez-Ferrer and H. Finkelmann, Soft Matter, 2013, 9, 4621-4627; (b) J. Garcia-Amoros, H. Finkelmann and D. Velasco, Chem. - Eur. J., 2011, 17, 6518-6523.

30 A. Sánchez-Ferrer, A. Merekalov and H. Finkelmann, Macromol. Rapid Commun., 2011, 32, 671-678. 
31 Y. Yu, M. Nakano and T. Ikeda, Nature, 2003, 425, 145.

32 S. Serak, N. Tabiryan, R. Vergara, T. J. White, R. A. Vaia and T. J. Bunning, Soft Matter, 2010, 6, 779-783.

33 M. Camacho-Lopez, H. Finkelmann, P. Palffy-Muhoray and M. Shelley, Nat. Mater., 2004, 3, 307-310.

34 Y. Yu, M. Nakano, A. Shishido, T. Shiono and T. Ikeda, Chem. Mater., 2004, 16, 1637-1643.

35 M. Yamada, M. Kondo, J. I. Mamiya, Y. Yu, M. Kinoshita, C. J. Barrett and T. Ikeda, Angew. Chem., Int. Ed., 2008, 47, 4986-4988.

36 S. Palagi, A. G. Mark, S. Y. Reigh, K. Melde, T. Qiu, H. Zeng, C. Parmeggiani, D. Martella, A. Sanchez-Castillo, N. Kapernaum, F. Giesselmann, D. S. Wiersma, E. Lauga and P. Fischer, Nat. Mater., 2016, 1-8.

37 C. L. van Oosten, C. W. M. Bastiaansen and D. J. Broer, Nat. Mater., 2009, 8, 677-682.
38 L. B. Braun, T. Hessberger, C. A. Serra and R. Zentel, Macromol. React. Eng., 2016, DOI: 10.1002/mren.201600015.

39 S. Zhang, B. Li, L. Tang, X. Wang, D. Liu and Q. Zhou, Polymer, 2001, 42, 7575-7582.

40 M. H. Li, P. Keller, B. Li, X. Wang and M. Brunet, Adv. Mater., 2003, 15, 569-572.

41 B. Li, S. Zhang, L. Tang and Q. Zhou, Polym. J., 2001, 33, 263-269.

42 C. Ohm, C. Serra and R. Zentel, Adv. Mater., 2009, 21, 4859-4862.

43 C. Ohm, E. K. Fleischmann, I. Kraus, C. Serra and R. Zentel, Adv. Funct. Mater., 2010, 20, 4314-4322.

44 D. L. Thomsen, P. Keller, J. Naciri, R. Pink, H. Jeon, D. Shenoy and B. R. Ratna, Macromolecules, 2001, 34, 5868-5875.

45 M.-H. Li, P. Auroy and P. Keller, Liq. Cryst., 2000, 27, 1497-1502.

46 X. Chen, L. Wang, Y. Chen, C. Li, G. Hou, X. Liu, X. Zhang, W. He and H. Yang, Chem. Commun., 2014, 50, 691-694. 